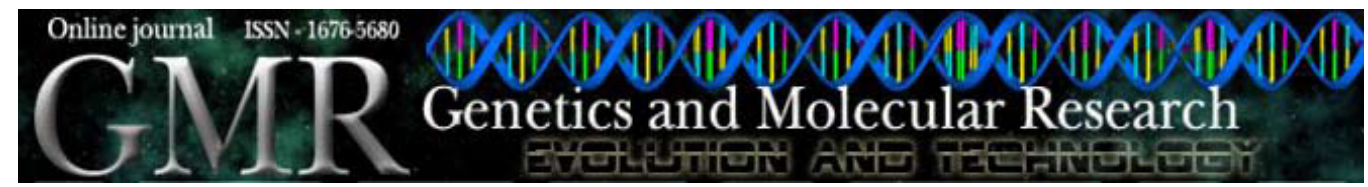

Case Report

\title{
Tetraploidization in Wilms tumor in an infant
}

\author{
E.T. Valera*, M.S. Brassesco*, C.A. Scrideli and L.G. Tone \\ Departamento de Puericultura e Pediatria, \\ Faculdade de Medicina de Ribeirão Preto, Universidade de São Paulo, \\ Ribeirão Preto, SP, Brasil
}

\author{
*These authors contributed equally to this study. \\ Corresponding author: E.T. Valera \\ E-mail: etvalera@hcrp.fmrp.usp.br
}

Genet. Mol. Res. 9 (3): 1577-1581 (2010)

Received May 14, 2010

Accepted July 1, 2010

Published August 10, 2010

DOI 10.4238/vol9-3gmr880

\begin{abstract}
Genetic instability is frequent in human cancer. Unscheduled tetraploidization can trigger cell transformation and tumorigenesis. We made a cytogenetic analysis by Giemsa-trypsin banding of a stage I, biphasic Wilms tumor diagnosed in a 10-month-old male. An evident karyotypic heterogeneity was found. Four different subclones of tumor cells were observed, with DNA content varying from diploid to near-tetraploid complements. The genetic events involved in the acquisition of aneuploidy in Wilms tumor remain unclear. We hypothesize that initial tetraploidization caused aberrant cell division, leading to abnormal chromosomal segregation, cell transformation and tumorigenesis.
\end{abstract}

Key words: Wilms tumor; Cancer biology; Cytogenetics; Tumor biology 


\section{INTRODUCTION}

Wilms tumor (WT), or nephroblastoma, is the most common malignant renal cancer to affect the pediatric population. Cytogenetic investigations on WT usually show nonrandom chromosomal changes characteristic of this tumor type. Numerical changes, mostly trisomies of chromosomes 7, 8, and 12, are particularly recurrent in this setting (Höglund et al., 2004).

Besides these structural cytogenetic observations, little is known about the genetic events involved in the acquisition of aneuploidy in WT. Changes in ploidy can result from several pathophysiological events, including aberrant polyploidization. Tetraploid cells represent an important intermediate on the route to aneuploidy and cancer. Unscheduled tetraploidy can arise through cell fusion, mitotic slippage or failure to undergo cytokinesis (Storchova and Kuffer, 2008).

In the present article, we describe the cytogenetic observation of tetraploidization and emergence of multiple subclones in WT occurring in an infant. Furthermore, the genetic implication of tetraploidization in human cancer, particularly in childhood WT, is also briefly reviewed.

\section{CASE REPORT}

An abdominal mass was palpable in a routine pediatric consultation of a 10-month-old male. This child was the first son of an apparently healthy and non-consanguineous couple. Family history disclosed no other cases of childhood cancer.

On physical examination, the child was in good general condition. He had a slightly distended abdomen and a mass was felt throughout his left flank and pelvis. Abdominal ultrasound (US) showed a bulky lesion, heterogeneous and arising from the left kidney. A computed tomography (CT) scan depicted the predominantly solid aspect of the lesion with intense, irregular enhancement following contrast injection. The mass measured $8 \mathrm{~cm}$ in length, while the contralateral right kidney was normal. Chest $\mathrm{CT}$ and bone scintigraphy were normal. The child was submitted to a US-guided True-Cut ${ }^{\mathbb{B}}$ biopsy. Histological examination of the specimen revealed a biphasic tumor composed of a predominant blastematous component, admixed with epithelial tubular structures, resembling immature renal tubules. The diagnosis of a stage I biphasic WT was established. The therapeutic approach followed the non-metastatic SIOP2001 protocol, which combines a four-week, two-drug, neo-adjuvant regimen (vincristine and dactinomycin), followed by surgical approach and adjuvant chemotherapy.

\section{CYTOGENETICS STUDIES}

A fresh pre-therapy WT sample (adjacent to areas of tumor verified by frozen section) was aseptically collected and minced with scissors on a Petri dish. The minced pieces were divided into T30 flasks and enzymatically disaggregated for $2 \mathrm{~h}$ on $0.5 \%$ collagenase type IV (Sigma Chemical Co., St. Louis, MO, USA) in DMEM-F10 medium (Gibco BRL, Life Technologies, Carlsbad, CA, USA) supplemented with $15 \%$ fetal bovine serum (FBS). Following disaggregation, the cells were centrifuged and the collagenase solution removed and replaced with medium. Cultures were harvested after 1 week with an overnight colcemid (Gibco) treatment at a final concentration of $0.25 \mu \mathrm{g} / \mathrm{mL}$. Cells were collected by trypsinization and centri- 
fuged at $1000 \mathrm{rpm}$. Trypsin activity was inhibited by resuspension in medium containing $10 \%$ FBS. The cells were pelleted by centrifugation, washed with phosphate-buffered saline, and resuspended in hypotonic medium $(0.075 \mathrm{M} \mathrm{KC} 1)$ for $20 \mathrm{~min}$ at $37^{\circ} \mathrm{C}$. Following hypotonic treatment, preparations were fixed three times with methanol:acetic acid (3:1). Further, cytogenetic analysis was performed by Giemsa-trypsin banding (GTG-banding).

\section{RESULTS}

Cytogenetic analysis of the WT sample showed karyotypic heterogeneity. Forty-one countable metaphase spreads were analyzed. Seventeen of these showed a normal complement of 46,XY while the rest showed near-diploid, near-triploid, and near-tetraploid chromosome numbers. Twelve metaphases $(29 \%)$ displayed $2 n=44-49$, five metaphases $(12 \%)$ showed $2 n=58-80$ and the other $17 \%$ were near-tetraploid with $2 n=81-93$ (Figure 1 ). No structural rearrangements were observed.

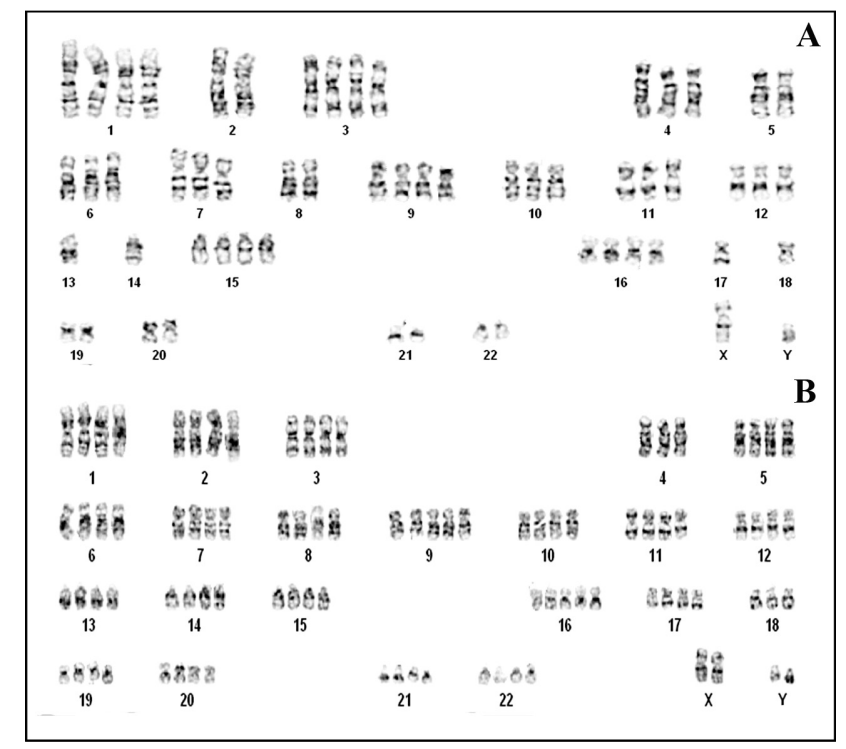

Figure 1. Partial results from conventional analysis of Giemsa-trypsin-banded Wilms tumor chromosome metaphase spreads. A. $58<3 n>$, XY, -?X, -?Y, +1, -2, +3, -5, -8, +9, -13, -13, -14, -14, +15, +16, -17, -17, -18, -18, $-19,-20,-21,-22$ karyotypes. B. $92, \mathrm{XXYY},-4,+9,+16,-18$ karyotypes.

\section{DISCUSSION}

Most malignant tumors usually display genetic instability, which can occur at the level of mutations in individual genes, by multiple structural and numerical aberrations or as largescale rearrangements with changes in cell ploidy. These genetic events may be responsible either for initiating or facilitating tumor progression (Storchova and Kuffer, 2008).

Wilms tumor is a malignant renal neoplasm derived from nephrogenic blastema. Besides the structural abnormalities that lead to homozygosity or hemizygosity for a small deletion on chromosome 11 (11p13) typically seen in WT, classical cytogenetic investigations 
have shown that this tumor usually carries numerical chromosomal changes. At least two cytogenetic pathways exist, one dominated by gains, with chromosomes 7, 8 and 12 trisomies being the most common abnormalities observed, and another characterized by losses of $1 \mathrm{p}, 16 \mathrm{q}, 11 \mathrm{p}$, 22q, and X (Höglund et al., 2004). However, despite the abundance of cytogenetic information, little is known about the genetic events involved in the acquisition of aneuploidy in WT.

In the present report, we describe the occurrence of WT in an infant. Tumor karyotype analysis revealed multiple neoplastic subclones, probably resulting from initial tetraploidization and abnormal chromosomal segregation.

Polyploid WT cases are relatively rare and have been estimated at only $8 \%$ of cases (Höglund et al., 2004). Even though a plethora of WT cases have been studied cytogenetically, no correlations between specific chromosome alterations and histologic subtypes have been found. Evidence for an association between a tetraploid DNA pattern and more aggressive malignancies with poor outcomes has been shown by flow cytometric analyses ( $\mathrm{Li}$ et al., 1995; Betts et al., 1997; Iyer et al., 1999). Also, it has been shown that intratumor DNA ploidy heterogeneity is significantly associated with unfavorable outcomes and death (Yildiz et al., 1994), reflecting the high genomic instability that leads to a rapid progression of the disease.

Tetraploidy is highly prevalent in different forms of cancer, suggesting a role for this cell cycle state in promoting cellular transformation. Aberrant polyploidization can arise through several mechanisms including cell fusion, endoreplication, DNA decatenation, mitotic slippage, or failure to undergo cytokinesis (Storchova and Pellman, 2004). Tetraploid cells represent an important intermediate on the route to aneuploid status and cancer. The major source of instability in tetraploid cells is the presence of supernumerary centrosomes that lead to the formation of multipolar spindles and consequently, aberrant chromosome segregation (Storchova and Pellman, 2004; Gisselsson et al., 2008). The tetraploid-intermediate model is also supported by the fact that the number of chromosomes in cancer cells is often very high, which is difficult to explain by a repeated accumulation of chromosomes at each division (Ganem et al., 2007). Furthermore, the presence of four copies of certain chromosomes, as seen for the aberrant neardiploid and near-triploid clones in the present case, points to the rational conclusion that chromosome heterogeneity may have been a consequence of random chromosome losses following tetraploidization. This genetic event seems to have facilitated the emergence of a WT in our little patient and may also be potentially implicated in tumorigenesis in other cases of infantile WT.

\section{Conflict of interest statement}

All authors have no conflict of interest to disclose.

\section{ACKNOWLEDGMENTS}

Research supported by Fundação de Amparo à Pesquisa do Estado de São Paulo (FAPESP Proc. \#06/04827-3 and \#07/54236-4).

\section{REFERENCES}

Betts DR, Koesters R, Pluss HJ and Niggli FK (1997). Routine karyotyping in Wilms tumor. Cancer Genet. Cytogenet. 96: 151-156.

Ganem NJ, Storchova Z and Pellman D (2007). Tetraploidy, aneuploidy and cancer. Curr. Opin. Genet. Dev. 17: 157-162. 
Gisselsson D, Hakanson U, Stoller P, Marti D, et al. (2008). When the genome plays dice: circumvention of the spindle assembly checkpoint and near-random chromosome segregation in multipolar cancer cell mitoses. PLoS One 3: e1871.

Höglund M, Gisselsson D, Hansen GB and Mitelman F (2004). Wilms tumors develop through two distinct karyotypic pathways. Cancer Genet. Cytogenet. 150: 9-15.

Iyer VK, Kapila K, Agarwala S, Dinda AK, et al. (1999). Wilms' tumor. Role of fine needle aspiration and DNA ploidy by image analysis in prognostication. Anal. Quant. Cytol. Histol. 21: 505-511.

Li P, Yokomori K, Tsuchida Y, Fujita M, et al. (1995). Flow cytometric nuclear DNA content analysis of renal tumors in children: prognostic significance of nuclear DNA ploidy. Tumour Biol. 16: 385-393.

Storchova Z and Pellman D (2004). From polyploidy to aneuploidy, genome instability and cancer. Nat. Rev. Mol. Cell Biol. 5: 45-54.

Storchova Z and Kuffer C (2008). The consequences of tetraploidy and aneuploidy. J. Cell Sci. 121: 3859-3866.

Yildiz I, Jaffe N, Aksoy F and el-Naggar AK (1994). Regional DNA content heterogeneity in Wilms' tumor: incidence and potential clinical relevance. Anticancer Res. 14: 1365-1369. 